\title{
MR of the Kidneys, Liver, and Spleen in Paroxysmal Nocturnal Hemoglobinuria
}

\author{
M. A. Roubidoux* \\ Department of Radiology, Duke University Medical Center, Durham, NC 27710, USA
}

Received: 21 May 1993/Accepted: 25 June 1993

\begin{abstract}
The magnetic resonance (MR) findings in the liver, kidneys, and spleen in eight patients with paroxysmal nocturnal hemoglobinuria (PNH) were retrospectively reviewed to determine whether characteristic features could be demonstrated. Eight patients underwent abdominal MR examinations by gradient echo sequences (seven patients), spin-echo sequences (seven patients), and inversion recovery (one patient). Signal intensities of the kidneys, liver, and spleen were visually evaluated. Autopsy and liver biopsy correlation were available in one case each. Renal signal intensity was decreased in all eight patients by either gradient-echo or T2-weighted sequences and in the single inversion recovery sequence. Hepatic signal intensity was decreased in three of eight patients on spin- and gradient-echo images. Splenic signal intensity was decreased in three of eight patients on spin- and gradient-echo images, and in two of these was manifest as focal low signal spots (Gamna-Gandy bodies). While the signal intensity in the renal cortex is typically decreased in patients with $\mathrm{PNH}$, signal intensities in the liver and spleen are variable. Low signal intensity in the kidneys is due to hemosiderin deposition resulting from intravascular hemolysis, whereas low signal intensity in the liver or spleen may be due to either transfusion siderosis, or as a consequence of hepatic or portal venous thrombosis.
\end{abstract}

Key words: Paroxysmal nocturnal hemoglobinuriaKidneys, MR studies-Liver, MR studies-Spleen, MR studies-Anemia.

* Present address: Department of Radiology, University of Michigan, 1500 E. Medical Center Drive, TC2910, Ann Arbor, MI 48109-0326, USA
Paroxysmal nocturnal hemoglobinuria (PNH) is an acquired hemolytic anemia in which membrane defects within the red cells make them susceptible to the lytic action of complement $[1,2]$. It is characterized by two major clinical features: chronic intravascular hemolysis with resulting gross hemoglobinuria and diffuse venous thrombosis $[2,3]$. Since the clinical course is variable, the diagnosis of PNH is often delayed or overlooked [2, 3]. When this diagnosis is considered, it can be confirmed by laboratory tests including the sucrose hemolysis test, urine examination for hemosiderin, and the Ham acid hemolysis test $[1,2]$.

Prior to magnetic resonance (MR) imaging, radiographic findings in $\mathrm{PNH}$ have been described as large kidneys with cortical infarcts, cortical thinning, or papillary necrosis $[4,5,7]$. The MR manifestations of iron deposition in the kidney, liver, and spleen in other hemolytic anemias, such as sickle cell disease and thalassemia, have been reported [6-9]. There have only been a few case reports of MR findings with spin-echo imaging in patients with $\mathrm{PNH}$, which describe low signal intensity in the renal cortex, due to the paramagnetic effects of iron deposition [10-12]. The findings in the kidneys, liver, and spleen on spin-echo, gradient-recalled echo, and inversion recovery sequences in eight additional patients are described.

\section{Materials and Methods}

Eight patients with PNH underwent MR examination. Five patients were female, three were male, with a mean age of 27 years. Diagnoses were made by laboratory studies and had already been established at the time of imaging in these patients for a mean of 7 years (range 215 years). All MR scans were obtained with a GE $1.5 \mathrm{~T}$ Signa Unit. The patients were referred for MRI because of clinically suspected abdominal venous thromboses. All examinations were reviewed retrospectively. 
Spin-echo sequences were obtained in the axial plane as follows: in six patients, T1-weighted images at TR 500, TE 11,15 , or 20; and in one patient at TR 600, TE 20. In five patients, T2-weighted images were obtained at TR 2000 or 2500 , TE 30 or 40 and 80 . All images were obtained at $7-\mathrm{mm}$ intervals with a matrix of $256 \times 128$ and 2 NEX. Respiratory compensation was used in all patients. One patient was imaged by an inversion recovery sequence at TR 2800 , TE 40 , IT 160. Axial gradient-echo (GRASS) images were obtained in seven patients at TR 33 or 37, TE 12 or 13 and at $30^{\circ}, 45^{\circ}$, or $60^{\circ}$ flip angles with $7-\mathrm{mm}$ intervals in the axial plane. In three patients, coronal images were obtained as follows: one by gradient echo of TR 33 and TE 13 , flip angle $45^{\circ}$, one at TR 37 , TE 12, flip angle $45^{\circ}$, and one at T1weighted images of TR 400, TE 11 . All of these were at 10-mm intervals.

The signal intensities of the liver and spleen were compared visually to skeletal muscle and categorized as either greater than that of muscle (normal), or equal to or less than that of muscle (decreased). Renal cortical signal intensity was visually compared to the renal medulla, and categorized as decreased, isointense, or increased.

\section{Results}

MR findings are summarized in Tables 1-3. The signal intensity in the renal cortex was decreased by either gradient-echo or T2-weighted sequences in all patients and decreased in the single inversion recovery sequence. In one patient with respiratory motion artifact, the kidney appeared of diffusely low signal on the gradient-echo sequence. Signal in the renal cortex was decreased compared to the medulla on $\mathrm{T} 1$ sequences in four of seven patients, isointense in two patients, and increased in one patient.

On spin- and gradient-echo sequences hepatic signal intensity was normal in five of eight patients. One patient (patient 3) had diffusely markedly diminished signal intensity. Two patients (4 and 8 ) had mildly decreased, inhomogeneous peripheral signal intensity.

Splenic signal intensity was normal by $\mathrm{T} 2$ or gradient-echo sequence in five of eight patients. Patient 3 had markedly low signal. Two patients ( 2 and 5 ) demonstrated focal distinct low signal dots in the spleen, but with otherwise normal splenic signal intensity.

Additional MR findings included hepatomegaly with marked narrowing of hepatic veins in patients 2 and 4 , and nonvisualization of the main hepatic veins in patient 8 . Superior mesenteric, portal vein, and vena cava thromboses were present in patient 5 .

Autopsy of patient 3 was performed within 1 week of MR imaging, and reported heavy iron deposition in the liver, spleen, and kidneys with centrilobular necrosis in the liver. Liver biopsy of patient 8 , performed within 1 month of MR imaging, reported central sinusoidal fibrosis consistent with hepatic vein thrombosis.

\section{Discussion}

Prior to the advent of MR imaging, there were no descriptions of $\mathrm{PNH}$ in the radiologic literature, due to the
Table 1. Signal intensities in the renal cortex

\begin{tabular}{ccclc}
\hline Patient & T1 & T2 & GRE & IR \\
\hline 1 & Decreased & Decreased & Decreased & Decreased \\
2 & Decreased & Decreased & Decreased & - \\
3 & Decreased & - & Decreased & - \\
4 & Decreased & - & Decreased & - \\
5 & Isointense & Decreased & Decreased & - \\
6 & Isointense & Decreased & - & - \\
7 & - & - & Decreased & - \\
8 & Increased & Decreased & Isointense & - \\
\hline
\end{tabular}

T1, T1-weighted; T2, T2-weighted; GRE, gradient-echo; IR, inversion recovery.

Table 2. Signal intensities in the liver

\begin{tabular}{clclc}
\hline Patient & T1 & T2 & GRE & IR \\
\hline 1 & Normal & Normal & Normal & Normal \\
2 & Normal & Normal & Normal & - \\
3 & Decreased & - & Decreased & - \\
4 & Decreased & - & Decreased & - \\
5 & Normal & Normal & Normal & - \\
6 & Normal & Normal & - & - \\
7 & - & - & Normal & - \\
8 & Decreased & Decreased & Decreased & - \\
\hline
\end{tabular}

See Table 1 for abbreviations.

Patient 3 had markedly decreased diffuse signal intensity.

Patients 4 and 8 had decreased inhomogeneous peripheral signal intensity.

Table 3. Signal intensities in the spleen

\begin{tabular}{clllc}
\hline Patient & T1 & T2 & GRE & IR \\
\hline 1 & Normal & Normal & Normal & Normal \\
2 & Normal & Decreased & Decreased & - \\
3 & Decreased & - & Decreased & - \\
4 & Normal & - & Normal & - \\
5 & Normal & Decreased & Decreased & - \\
6 & Normal & Normal & - & - \\
7 & - & - & Normal & - \\
8 & Normal & Normal & Normal & - \\
\hline
\end{tabular}

See Table 1 for abbreviations.

Patients 2 and 5 demonstrated focal decreased signal intensity spots.

lack of characteristic radiologic abnormalities. Furthermore, contrast material is usually contraindicated, not only because renal insufficiency often occurs, but also because complement activation may induce or exacerbate a hemolytic crisis. Abdominal thromboses often occur in $\mathrm{PNH}$, requiring prompt, minimally invasive diagnosis, and vigorous treatment [13-15]. Since MR imaging can detect vascular thromboses without contrast material, MR imaging may be extremely helpful in this disease.

Only a few cases of the MR findings in patients with PNH have been reported. These visually com- 

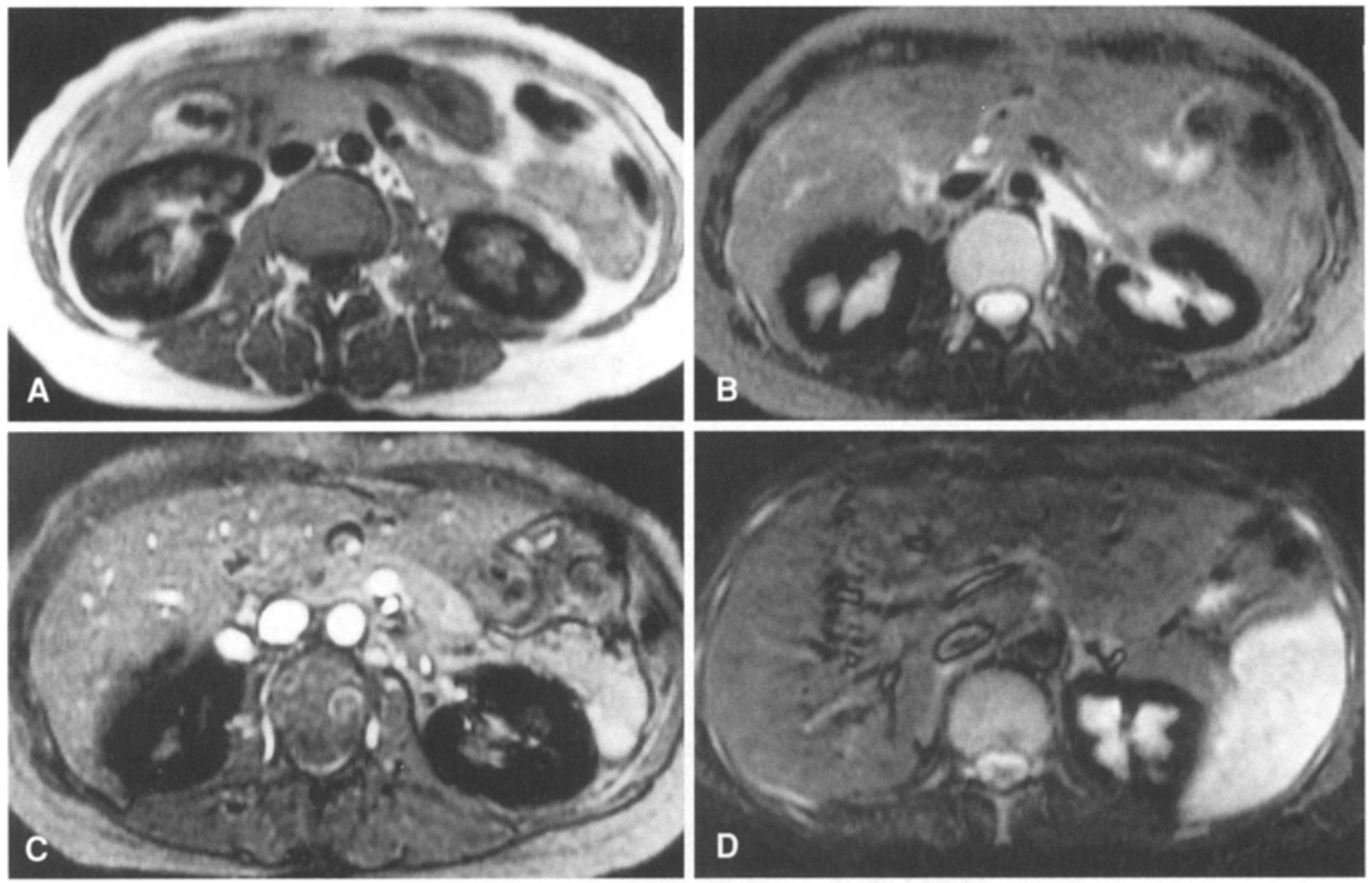

Fig. 1. In PNH, low renal cortical signal from hemosiderin deposition may be seen on all pulse sequences. This effect is greater with $\mathrm{T} 2$ or gradient-echo sequences. A T1-weighted SE image (500/15); (B) T2weighted SE image (2500/80); (C) GRASS image (33/13/60 $)$; (D) inversion recovery image $(2800 / 40 / 160)$.

pared the renal cortex to the medulla and described low renal cortical signal intensity on $\mathrm{T} 1$ - and $\mathrm{T} 2$-weighted sequences $[10-12,16]$. The signal intensity of the liver has been described in two cases as normal and in one case as decreased $[11,12,16]$. The signal intensity of the spleen was reported to be normal in a single case [16]. Visual comparison of hepatic and splenic signal intensity with paraspinous musculature, reported to be useful in assessment of parenchymal iron deposition in other diseases, was used to evaluate these organs in this study [16].

The MR findings in the kidney in these eight patients confirm case reports of low signal in the renal cortex on T1- and T2-weighted sequences, and also demonstrate this effect on gradient-echo and inversion recovery sequences (Fig. 1).

The signal intensity of the normal kidney on T1weighted images is low, with the renal cortex slightly more intense than the medulla, resulting in corticomedullary differentiation [7]. This normal appearance was seen in one of the seven patients. The cortex may become isointense to the medulla on T1-weighted images in dehydration and a variety of renal disorders $[17,18]$.
This finding was demonstrated in two of seven patients. Decreased signal intensity in the cortex, as described in the prior case reports of $\mathrm{PNH}$, was seen in four of seven patients.

On T2-weighted images, the cortex and medulla both have high signal intensity, similar to the signal intensity of the spleen and fat, and there is no visual distinction between the cortex and medulla $[17,18]$. In all eight patients, low signal in the cortex was identified by $\mathrm{T} 2$ or gradient-echo sequences. This low signal in PNH is due to intense hemosiderin deposition in the renal cortex, the characteristic pathologic feature of this disease [19]. The ferric iron in hemosiderin is a paramagnetic agent which causes both decreased $\mathrm{T} 1$ and $\mathrm{T} 2$ relaxation times of the nearby hydrogen atoms. The extreme shortening of the T2 relaxation time decreases signal in both $\mathrm{T} 1$ and $\mathrm{T} 2$ images, but this effect is greater in T2-weighted sequences [20, 21] (Fig. 2). This low cortical signal is also more conspicuous on T2-weighted images due to the adjacent high signal medulla (Figs. 2 and 3). Gradient-echo imaging is also sensitive to T2 signal loss resulting from dephasing caused by the field inhomogeneities induced by paramagnetic agents, particularly because it lacks the $180^{\circ}$ refocusing pulse, which partially recovers signal loss in spin-echo imaging caused by field inhomogeneities [20,21].

The intense iron deposition in the proximal tubule cells in the renal cortex characteristic of this disease is related to the location and the mechanism of hemolysis (Fig. 4). Most hemolytic anemias, such as sickle cell 

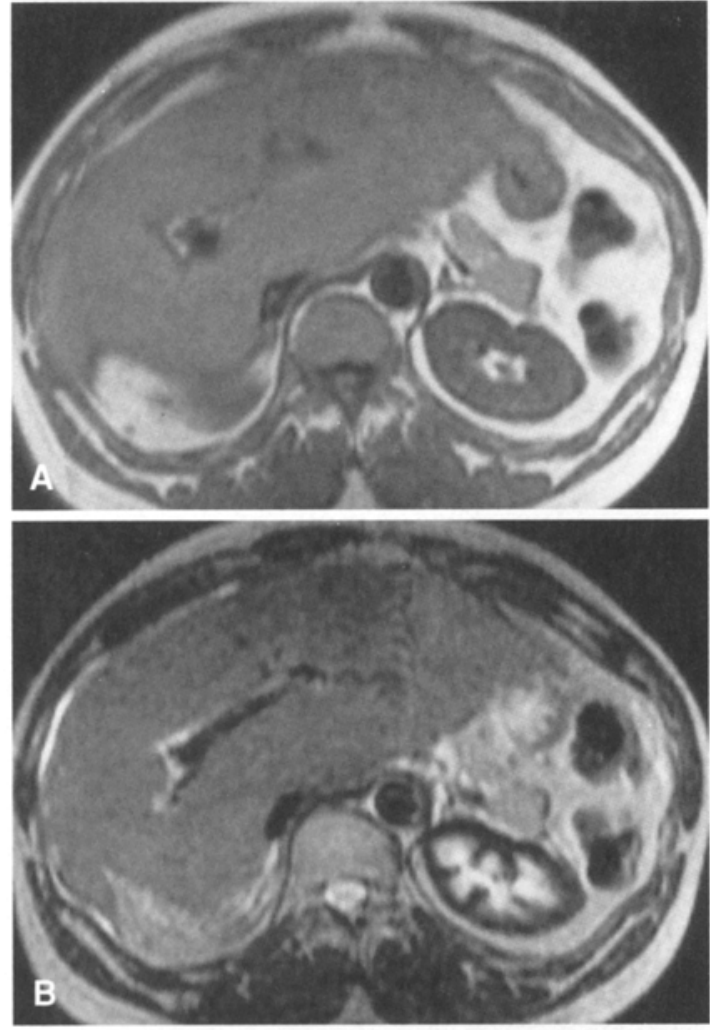

Fig. 2. In some patients with $\mathrm{PNH}$, the low cortical signal is more apparent on T2-weighted SE images. A T1-weighted SE image (500/ 11); (B) T2-weighted SE image (2500/80).

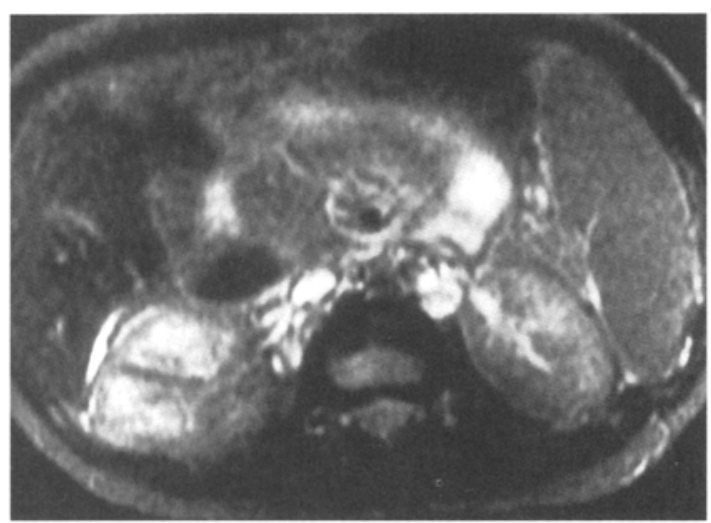

Fig. 3. A more subtle decrease in renal cortical signal in a 13 -yearold patient. T2-weighted SE image (2500/80).

disease, autoimmune hemolytic anemia, or hereditary spherocytosis, are characterized by extravascular hemolysis (i.e., reticuloendothelial degradation of damaged or defective red cells) resulting in iron deposition in the liver and spleen [22, 23]. In contrast, intravascular hemolysis which occurs in either PNH or in mechanical hemolysis due to prosthetic cardiac valves, results in direct hemoglobin release into the plasma [24, 25]. At very low rates of intravascular hemolysis, the hemoglobin attaches to the plasma protein haptoglobin and is removed by the hepatocytes. When plasma haptoglobin becomes saturated with hemoglobin, free hemoglobin passes through the glomerulus, is reabsorbed in the proximal tubules, and stored as hemosiderin $[2,19,26$, 27]. When the tubular absorptive capacity is exceeded, this filtered hemoglobin is lost in the urine [27]. Interestingly, renal function is often abnormal in these patients due to venous microthrombi rather than to tubular damage from hemosiderin deposition $[4,5]$.

Renal iron deposition in PNH is unrelated to transfusion therapy, since patients with transfusional siderosis and primary hemochromatosis have normal renal signal intensity. This is consistent with pathologic reports which demonstrate that little renal iron deposition occurs in the latter two entities $[7,16,28-30]$.

Low renal cortical signal intensity from iron deposition is characteristic but not pathognomonic of $\mathrm{PNH}$, since this finding is also reported in sickle cell disease [7]. This may be due to episodes of hemolytic crises, resulting in some degree of intravascular hemolysis and renal hemosiderin deposition, or due to iron deposition in the glomerulus, although, pathologically, less renal iron is detected in sickle cell disease than in PNH [22, $26,28]$. Low renal medullary signal due to hemorrhage has been described in hemorrhagic fever with renal syndrome, representing a pattern of findings on MR opposite to those seen in PNH [31].

In the liver, patients with PNH typically have decreased or absent parenchymal iron (due to heavy renal hemoglobin deposition and urinary iron loss) unless multiple transfusions have been given $[24,28,32,33]$. This is in contrast to other chronic hemolytic anemias which have abnormally increased hepatic and splenic iron deposition, due to reticuloendothelial (i.e., extravascular) red cell destruction $[23,25]$. It is therefore unlikely that the decreased hepatic signal in three patients was caused by iron deposition resulting from the intravascular hemolysis in PNH. The diffuse very low signal intensity in the liver and spleen demonstrated in patient 3 is consistent with previous MR findings in transfusional siderosis, and was most likely due to the history of 15 years of multiple transfusions (Fig. 5) [16]. Autopsy of this patient demonstrated diffuse intense iron deposition consistent with transfusional siderosis, in addition to centrilobular necrosis due to hepatovenoocclusive disease.

An additional finding in the liver in PNH is hepatic venous thrombosis, a life-threatening complication. This typically involves at least the secondary and tertiary venous radicals (whether or not the primary veins are also involved) and is caused by thrombi consisting of red blood cell ghosts [14]. Centrilobular necrosis and hepatic congestion occur secondary to this thrombotic 

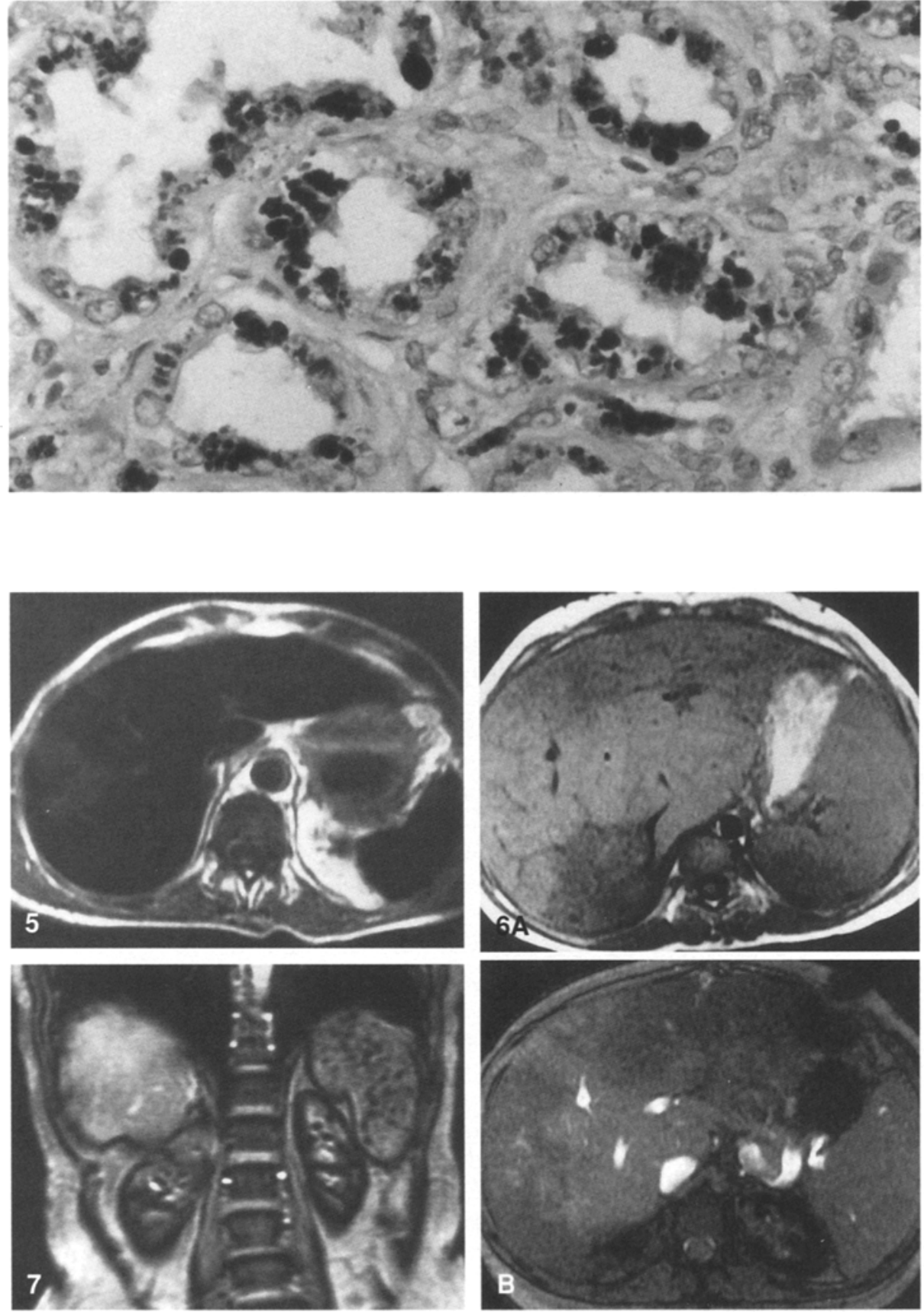

Fig. 4. Hemosiderin fills proximal tubule cells (hematoxylin \& eosin stain; original magnification, $\times 250$ ).
Fig. 5. Very low signal in liver and spleen demonstrated in this patient with PNH and a 15-year history of transfusions, resulting in reticuloendothelial iron deposition. T1weighted image $(500 / 20)$.

Fig. 6. Decreased peripheral signal, hepatomegaly, and narrowing of vasculature in two patients with hepatovenoocclusive disease. A Patient 8 , axial $\mathrm{T} 1$-weighted image (500/15); (B) patient 3, axial GRASS image $\left(37 / 12 / 45^{\circ}\right)$.

Fig. 7. Gamna-Gandy bodies in the spleen in patient with chronic portal vein thrombosis due to $\mathrm{PNH}$. Coronal GRASS image (33/13/ $30^{\circ}$ ). process. Older lesions may demonstrate confluent fibrosis $[13,14]$. Decreased inhomogeneous hepatic signal in patients 4 and 8 was accompanied by hepatomegaly and narrowing or nonvisualization of the intrahepatic veins, and these findings are likely due to hepatovenoocclusive disease (Fig. 6). A similarly inhomogenous pattern was demonstrated by contrast-enhanced CT in patient 4, and hepatovenoocclusive disease was confirmed by biopsy in patient 8 . Similar MR and CT findings have been described in hepatic venous thrombosis [34-36].
The spleen contributes little to the intravascular hemolytic process in PNH. Therefore, the spleen is neither enlarged or congested, and erythrophagocytosis is absent, unlike the spleens in patients with thalassemia, sickle cell disease, or hereditary spherocytosis. Like the liver in $\mathrm{PNH}$, iron is characteristically absent unless transfusional siderosis has occurred [24].

Normal splenic intensity on MR is visually similar to hepatic signal intensity on T1-weighted images. On T2-weighted images the signal intensity is very bright, near fat, or that of the kidney $[9,18]$. Abnormally de- 
creased splenic signal intensity has been previously described in patients with transfusional siderosis, hemochromatosis, and sickle cell disease consistent with iron deposition $[6,9,16]$. The diffuse low signal intensity in both the spleen and liver in patient 3 is probably due to transfusion siderosis.

Focal low signal intensity spots in the spleen were identified on T2 and gradient-echo imaging in two patients with $\mathrm{PNH}$, one of whom also demonstrated evidence of chronic portal vein thrombosis. This finding is most clearly demonstrated on gradient-echo images, likely secondary to the breath-hold technique used in this sequence (Fig. 7). These low intensity foci have been seen in patients with portal hypertension or portal vein thrombosis, and are considered to represent siderotic nodules or Gamna-Gandy bodies, organized foci of hemorrhage, hemosiderin deposition, and fibrosis. These nodules generally are not visualized by CT, although have been reported as hyperechoic lesions on ultrasound $[37,38]$.

In summary, in PNH, iron deposition in the kidneys, liver, and spleen is readily demonstrated by MR imaging, particularly by $\mathrm{T} 2$ and gradient-echo sequences. Signal intensity in the renal cortex is markedly decreased, due to the heavy deposition of hemosiderin in the proximal tubules resulting from intravascular hemolysis. The signal intensity in the liver and spleen is normal unless transfusional siderosis or vascular thrombosis have occurred.

\section{References}

1. Williams WJ, Beutler E, Erslev AJ, Lichtman MA. Hematology, 4th ed. New York: McGraw Hill, 1990:188-192

2. Wintrobe MM. Clinical hematology, 8th ed. Philadelphia: Lea and Febiger, 1981:171-191

3. Formon K, Sokol J, Hewit S, Stamps BK. Paroxysmal nocturnal emoglobinuria. Ser Hematol 1984;71:217-226

4. Braren V, Butler SA, Hartman RC, Jenkins DE Jr. Urologic manifestations of paroxysmal nocturnal hemoglobinuria. J Urol 1975; 114:430-434

5. Clark DA, Butler SA, Varen V, Hartmann RC, Jenkins DE. The kidneys in paroxysmal nocturnal hemoglobinuria. Blood 1981; $57: 83-89$

6. Brasch HC, Wesbey GE, Gooding CA, Koerper MA. Magnetic resonance imaging of transfusional hemosiderosis complicating thalassemia major. Radiology 1984;150:767-771

7. Lande IM, Glazer GM, Sarnaik S, Aisen A, Rucknagel D, Martel W. Sickle-cell nephropathy: MR imaging. Radiology 1986;158:379-383

8. Gormori JM, Grossman RI, Drott HR. MR relaxation times and iron content of thalassemic spleens: an in vitro study. $A J R$ 1988;150:567-569

9. Adler DD, Glazer GM, Aisen AM. MRI of the spleen: normal appearance and findings in sickle-cell anemia. AJR 1986;147: 843-845

10. Mulopulos GP, Turner DA, Schwartz MM, Murakami ME, Clark JW. MRI of the kidneys in paroxysmal nocturnal hemoglobinuria. AJR 1986;146:51-52

11. Lupetin AR. Magnetic resonance appearance of the kidneys in paroxysmal nocturnal hemoglobinuria. Urol Radiol 1986;8:101-103

12. Kim SH, Han MC, Lee JS, Kim S. Paroxysmal nocturnal hemoglobinuria. Acta Radiol 1991;32:315-316
13. Hartman RC, Luther AB, Jenkins DE, Tenorio LE, Saba HI. Fulminant hepatovenous thrombosis (Budd-Chiari syndrome) in paroxysmal nocturnal hemoglobinuria: definition of a medical emergency. John Hopkins Med J 1980;146:247-254

14. Peytremann R, Rhodes RS, Hartman RC. Thrombosis in paroxysmal nocturnal hemoglobinuria (PNH) with particular reference to progressive, diffuse hepatic venous thrombosis. Ser Hamatol 1972;5:115-136

15. Haddad MC, Clark DC, Sharif HS, Shahed MA, Aideyan O, Sammak BM. MR, CT, and ultrasonography of splanchnic venous thrombosis. Gastrointest Radiol 1992;17:34-40

16. Siegleman ES, Mitchell DG, Rubin R, et al. Parenchymal versus reticuloendothelial iron overload in the liver: distinction with $M R$ imaging. Radiology 1991;179:361-366

17. Glazer GM. MR imaging of the liver, kidneys, and adrenal glands. Radiology 1988; 166:303-312

18. Ehman RL, Kjos BO, Hricak H, Brasch RC, Higgins CB. Relative intensity of abdominal organs in MR images. JCAT 1985;9:315-319

19. Hutt MP, Reger JF, Neustein HB. Renal pathology in paroxysmal nocturnal hemoglobinuria. An electron microscopic illustration of the formation and disposition of ferritin in the nephron. Am J Med 1961;31:736-746

20. Stark DD. Hepatic iron overload: paramagnetic pathology. $R a-$ diology 1991;179:333-335

21. Hernandez RJ, Samaik SA, Lande I, et al. MR evaluation of liver iron overload. JCAT 1988;12:91-94

22. Crosby WH, Dameshek W. The significance of hemoglobinemia and associated hemosiderinuria, with particular reference to various types of hemolytic anemia. J Lab Clin Med 1951;38:829-841

23. Sears DA, Anderson PR, Foy AL, William HL, Crosby WH. Urinary iron excretion and renal metabolism of hemoglobin in hemolytic diseases. Blood 1966;28:708-725

24. Crosby WH. Paroxysmal nocturnal hemoglobinuria. Blood 1953;8:769-777

25. Sigler AT, Forman EN, Zinkham WH, Neill CA. Severe intravascular hemolysis following surgical repair of endocardial cushion defects. Am J Med 1963;35:467-479

26. Bunn HF, Eshman W, Bull RW. The renal handling of hemoglobin. I Glomerular filtration. J Exp Med 1969;129:909

27. Bunn HF, Jandi JH. The renal handling of hemoglobin. $J$ Exp Med 1969;129:925

28. Leonardi P, Ruol PL. Renal hemosiderosis in the hemolytic anemias: diagnosis by means of needle biopsy. Blood 1960;16:1029-1038

29. Cappell DF, Hutchison HE, Jowett M. Transfusional siderosis: the effects of excessive iron deposits on the tissues. $J$ Pathol Bacteriol 1957;674:245-261

30. Sheldon JH. Haemochromatosis. London: Oxford University Press, 1935

31. Kim SH, Kim S, Lee JS, et al. Hemorrhagic fever with renal syndrome: MR imaging of the kidney. Radiology 1990;175:823-825

32. Heitzman EJ, Campbell JS, Stefanini M. Paroxysmal nocturnal hemoglobinuria with hemosiderin nephrosis. Am I Clin Pathol 1953;23:975-985

33. Hartman RC, Jenkins DE, McKee C, Heyssel RM. Paroxysmal nocturnal hemoglobinuria: clinical and laboratory studies relating to iron metabolism and therapy with androgen and iron. Medicine 1966;331:363

34. Murphy FB, Steinberg HV, Shires GT III, Martin LG, Bernardino ME. The Budd-Chiari syndrome: a review. AJR 1986;147:9-15

35. Stark DD, Hahn PF, Trey C, Clouse ME, Ferrucci JT Jr. MRI of the Budd-Chiari syndrome. AJR 1986;146:1141-1148

36. Mitchell DG. Focal manifestations of diffuse liver disease at MR imaging. Radiology 1992;185:1-11

37. Minami M, Itai $Y$, Ohtomo $\mathrm{K}$, et al. Siderotic nodules in the spleen: MR imaging of portal hypertension. Radiology 1989;172: $681-684$

38. Sagoh T, Itoh K, Togashi K, et al. Gamna-Gandy bodies of the spleen: evaluation with MR imaging. Radiology 1989;172:685-687. 